\title{
A Theoretical Study of Mufflers with Open-ended Intruding Perforated Tubes
}

\author{
Chao-Nan Wang \\ Department of Engineering Science and Ocean Engineering, National Taiwan University, Taipei, Taiwan 106, R.O.C.
}

\author{
Malcolm J. Crocker ${ }^{\dagger}$ \\ Department of Mechanical Engineering, Auburn University, AL 36849, USA
}

(Received 1 January 2001; revised 12 October 2001; accepted 22 July 2002)

\begin{abstract}
This paper presents an analysis of the performance of an open-ended intruding perforated-tube muffler. A theoretical formulation of the transfer matrix for such a muffler is given. The derivation is based on the assumption of plane wave propagation. By using continuity and momentum equations and the equation of isentropicity at the open end, the transfer matrix of the inlet open-end intruding tube can be derived. Then the transfer matrix and also the transmission loss of the whole muffler can be evaluated. The predicted results are shown to agree well with the experimental measurements presented in the literature. Finally, the effects of the mean flow velocity, porosity, and length of the intruding perforated-tube are investigated analytically.
\end{abstract}

${ }^{\dagger}$ Member of the International Institute of Acoustics and Vibration (IIAV)

\section{INTRODUCTION}

Mufflers are used widely in reducing the noise generated by vehicles and machines. Many theoretical and experimental studies have been made to analyse the performance of different types of muffler components, such as simple expansion chambers, plug mufflers and concentric-tube resonators. It is evident that perforated tubes are commonly-used components in exhaust muffler systems. The analysis for the perforated tube muffler components is normally based upon the coupled equations derived by Sullivan and Crocker ${ }^{1}$. Munjal et al. ${ }^{2}$ proposed a generalised de-coupling method to solve the coupled equations. The method works well, although instability occurs in the peak attenuation region. Peat ${ }^{3}$ has converted this approach into an eigen value problem called the numerical de-coupling method and has overcome the instability problem. However, only components, such as concentric-tube resonators and plug mufflers, have been analysed with this method. Open-end intruding perforated tube muffler components have still not been analysed since it is not easy to specify the boundary condition at the open end.

In 1982, Thawani and Noreen ${ }^{4}$ investigated many types of muffler elements that are commonly used in muffler designs. However, for mufflers with open-ended intruding perforated tubes, they did not specify the boundary condition for the open end in their paper. Eriksson and Thawani ${ }^{5}$ also studied the same types of muffler, however, they only published their experimental results. Then Luo et al. ${ }^{6}$ proposed a method that specifies the boundary condition at the end of the perforated tube. They derived the transfer matrices between the inlet and outlet of the inner perforated tube and between the inlet of the inner tube and the outlet of the outer tube respectively. The mean flow velocity was not considered in their paper and also rather erratic results were obtained from their theoretical approach. In 1995, $\mathrm{Wang}^{7}$ analysed the open-ended intruding perforated tube muffler component using the boundary element method. In his study the mean flow veloc- ity was not included in the governing equation. The effect of the mean flow velocity was included only in the impedance of the perforated tube. Recently, Gogate and Munjal ${ }^{8}$ and Munjal et al..$^{9,10}$ have proposed the concept of stagnation pressure and derived the transfer matrix for open-ended threeduct perforated elements and reverse-flow, three-duct, openended perforated element mufflers (Fig. 1). This approach works well. However, there are still some other muffler elements that have not yet been modelled.

In this study, we deal with one of these elements, as shown in Fig. 2. First, the transfer matrix of a straight-through intruding perforated tube component is derived. Then the combined transfer matrix of the whole muffler is evaluated and subsequently the transmission loss is obtained. The theoretical solution is compared with experimental measurements presented in the literature. Then the influence of the mean flow velocity, porosity, and length of the perforated tube is also investigated.

\section{DERIVATION OF THE TRANSFER MATRIX}

Figure 2(b) shows the geometry of the inlet part of the open-ended intruding tube muffler. The purpose of this section is to present a derivation of the transfer matrix for this component, that is, the duct segment between points 1 and 5 . For the points 1 to 4 , a two-duct perforated segment is formed which results in coupled wave equations. Since the air flow grazes along the surface of the centre tube, the mean flow velocity in the outer expansion duct is small and is assumed to be zero. Therefore, the continuity and momentum equations for each duct are given by:

1) For the centre tube:

$$
\begin{gathered}
\rho_{0} \frac{\partial u_{c}}{\partial x}+V \frac{\partial \rho_{c}}{\partial x}+\frac{4}{D_{1}} \rho_{0} w+\frac{\partial \rho_{c}}{\partial t}=0, \\
\rho_{0}\left(\frac{\partial}{\partial t}+V \frac{\partial}{\partial x}\right) u_{c}+\frac{\partial \rho_{c}}{\partial x}=0 .
\end{gathered}
$$

